\title{
Fast-Response Liquid Crystal Lens Doped with Multi-Walled Carbon Nanotubes
}

\author{
Hui LI ${ }^{1,2,4}{ }^{*}$, Fan PAN ${ }^{3}$, Yuntao WU ${ }^{1,2}$, Yanduo ZHANG ${ }^{1,2}$, Xiaolin XIE $^{4}$ \\ ${ }^{1}$ School of Computer Science and Engineering, Wuhan Institute of Technology, Wuhan 40073, P. R. China \\ ${ }^{2}$ Hubei Key Laboratory of Intelligent Robot, Wuhan 40073, P. R. China \\ ${ }^{3}$ College of Post and Telecommunication, Wuhan Institute of Technology, Wuhan 40073, P. R. China \\ ${ }^{4}$ School of Chemistry and Chemical Engineering, Huazhong University of Science and Technology, Wuhan 40073, P. R. \\ China \\ cross $^{\text {ref }}$ http://dx.doi.org/10.5755/j01.ms.22.2.12913
}

Received 20 August 2015; accepted 05 February 2016

In this paper, a relatively fast-response liquid crystal (LC) lens was proposed, which was fabricated by a simple method. Multi-walled carbon nanotubes (MWCNTs) were utilized in fabricating the LC lens. As MWCNTs were doped into the LCs, the dielectric anisotropy of the mixture changed, which was the key factor in solving the technical barrier of slow response time. In experiments, the effects of doping with MWCNTs were demonstrated. The concentration of doped MWCNTs was discussed in detail, and the best concentration and doping method were analyzed. The relationship between the concentration and response time was also obtained. This LC lens had a sub-millisecond response time, which was a relatively fast response time in comparison to conventional LC lenses of pristine LCs. Thus, this proposed method could be considered as a new method to realize fast-response LC lens.

Keywords: liquid crystal material, doped multi-walled carbon nanotubes, fast response, lens design, tunable focal length.

\section{INTRODUCTION}

The birefringence of liquid crystals (LCs) is well known, and its electrically tunable birefringence has been utilized to fabricate different LC products, such as LC lenses [1,2], LC spatial light modulators [3,4], LC displays [5], and LC Fabry-Perot devices [6,7]. Many studies have been conducted on how to utilize LCs for fabricating imaging optical lenses $[8,9]$. Owing to its tunable focusing, compactness, portability, and easy integration with other optical systems, LC lenses, in many imaging applications, can be an effective alternative to other traditional optical lenses. However, there are some difficulties when fabricating high quality LC lenses such as the slow response time. In order to solve that problem, the current work is aimed to change the structure of LC lens, which increases the manufacturing costs and the fabrication process complexity $[8,9]$. However, there are only a few works on the inside doped material of the LC lens. Thus, the research presented considers doping of LC material by carbon nanotubes, which will be a relatively simple method to solve that problem. As is known, a CNT is a tube-shaped material made of carbon that has a diameter measured on the nanometer scale. Overall, CNTs show a unique combination of stiffness, strength, and tenacity compared to other fiber materials, which usually lack one or more of these properties. Thermal and electrical conductivity are also much higher compared to other conductive materials [10]. Recently, the refractive index and other optical properties of LCs doped with CNT have been investigated $[11,12]$, as well as the refractive index of LCs doped with MWCNT [13]. According to the

\footnotetext{
${ }^{*}$ Corresponding author. Tel.: +86-27-87992007; fax: +86-27-87992007

E-mail address: lihui00317@163.com (H. Li)
}

research results, in theory, a LC lens doped with MWCNT in principle can be achieved.

In this paper, MWCNTs were used to fabricate LC lenses. To best of our knowledge, this LC lens doped with CNT is first proposed. The purpose of this work is to represent the fabrication method and the basic electricoptical features of the LC lens. The mechanism of its performance improvement will be also preliminarily discussed and analyzed. The relatively fast response time, simple fabrication process, and the capability of massive production at low cost associated with the proposed LC lens would make it an attractive candidate over the conventional LC lens. This study showed that the focusing time for a specification doped with $0.02 \%$ MWCNT can be reduced to sub-milliseconds, and the applied voltage drops significantly to less than $5 \mathrm{~V}$.

\section{EXPERIMENTAL PROCEDURES}

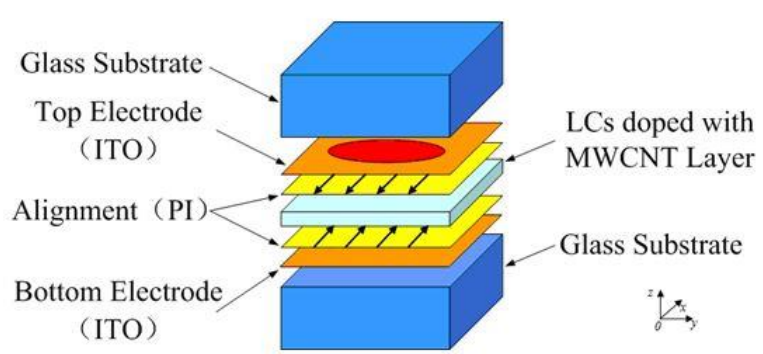

Fig. 1. Device structure of the proposed LC lens [14]

A cross profile of this mentioned LC lens, which primarily consisted of a LC layer doped with MWCNTs and two indium tin oxide (ITO) substrates, is depicted in Fig. 1.

The technological flow for the fabrication of the LC lens was as follows. UV-photolithography and wet 
hydrochloric acid (hereafter referred as HCL) etching on the top ITO film were used to fabricate only one roundhole electrode pattern whose diameter is $2.0 \mathrm{~mm}$. Coated both internal surfaces of those two substrates and rubbed along the $x$ direction, consequently a thin film of polyimide (PI), which forms an alignment layer, was made. As shown in Fig. 1, this allowed all the LC directors to produce about a $2^{\circ}$ pre-tilt angle. After the above procedures, the two substrates with expected electrode patterns and the formed alignment films composed the empty LC cell by sandwiching glass micro-spheres as the spacer. The most important fabrication step was to uniformly dope a trace of MWCNT into the LC materials with as little reunion as possible. In this paper, MWCNTs were obtained from Shenzhen Nanotech Port Co. Ltd, which had the main features of $>90 \mathrm{wt} \%$, length of $1-2 \mu \mathrm{m}$, and outside diameter of $10-20 \mathrm{~nm}$. An appropriate amount of MWCNTs was pulled into a small amount of alcohol, and was continually shocked by an ultrasonic shaker for $3.5 \mathrm{~h}$. (The specific amounts that obtained the best results will be discussed in detail later.) Then, the processed mixtures were mixed with commercially available LCs, E7 (Merck), in a clean glass-tube. The mixtures of LCs and MWCNTs were further mixed by a test tube shaker for $30 \mathrm{~min}$ at $1000 \mathrm{rmp} / \mathrm{m}$. To volatilize the alcohol, the whole materials were placed in an oven at around $100{ }^{\circ} \mathrm{C}$ for $30 \mathrm{~min}$. The previous steps were repeated three more times to maximize the evaporation of the remaining alcohol. During those repeats, the shock time of the test tube shaker was set at $1.5 \mathrm{~h}$ for a good dispersion. After the relatively uniform LC materials doped with WMCNTs were achieved, the materials, controlled at $80{ }^{\circ} \mathrm{C}$ were injected into the empty cell by capillary action. While cooled to room temperature slowly, the LC cell was sealed by AB glues. According to other critical technological parameters depicted for the LC lens, $100 \mu \mathrm{m}$ diameter microspheres of spacers was used to fabricate an empty LC cell, and those specific values of operation voltage will be given in the following section.

\section{RESULTS AND DISCUSSION}

As it is known, LC is a material of birefringence, playing a key role for the photorefractive feature of the LC lens. According to the elastic continuum theory, each single rotating tilt angle of the LC director presents a refractive index value in the LC layer. As the single circular-hole electrode pattern is on the top substrate, there will be different rotating tilt angles in the LC layer, which form a gradient refractive index distribution. Thus, the incident polarized light should be focused when the refractive index profile has a parabolic curve as expectation. According to both geometrical optics and elastic continuum theory, the focal length of the LC lens is $[8,9]$ :

$$
f=\frac{r^{2}}{2 \Delta n d_{L C}},
$$

where $f$ is the focal length, $r$ is the radius of the circularhole, $d_{L C}$ is the cell thickness, and $\Delta n$ is the difference between the centre and the margin of the aperture.

Due to many unique physical, chemical, and electronic properties, CNTs can be widely used as guest dopants and could promote the electro-optical characterizations of the LC lens. And LCs doped with CNTs exhibit many advantages for developing novel optoelectronic devices.

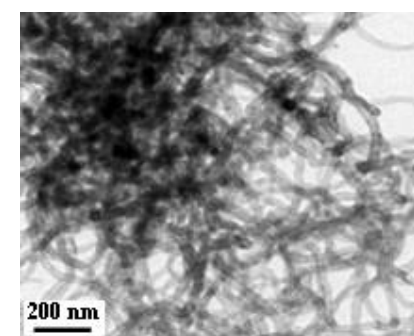

a

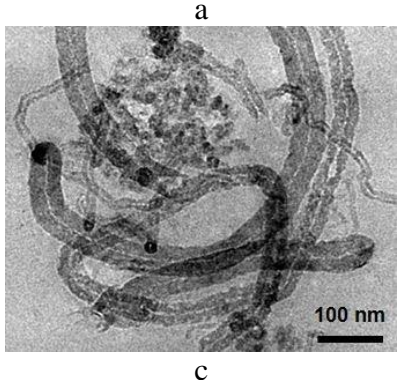

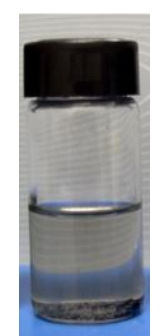

b

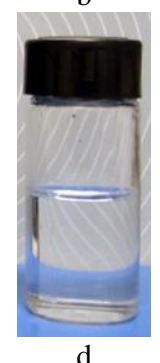

Fig. 2. Experimental images of LCs doped with MWCNTs about 0.02 wt.\%: a-SEM image without alcohol; b-camera image without alcohol after three days; c-SEM image with alcohol; d-camera image with alcohol after three days

However, applications of CNTs still face some difficulties such as infusibility, indissolubility, conglomeration, and entanglement random array. Because of these problems, MWCNTs don't disperse well in LCs, which seriously affects the features of the mixture. Generally, the usual solutions are chemically synthesized to solve such problems. But, this type of chemical reaction is a complicated method and the cost is high. Thus, a simple method using alcohol to uniformly disperse MWCNTs into LCs was proposed in this paper. Alcohol was chosen as the dispersing solvent because it effectively enabled the MWCNTs to uniformly disperse in the LCs. Alcohol also has very good volatility, and can be easily processed. The comparative-test images are presented in Fig. 2, which demonstrates the assumptions. The LCs doped with MWCNTs, as shown in Fig. 2 a, exhibited obvious agglomeration, indicating that the MWCNTs didn't disperse well in the LCs. After three days, there was an apparent stratification in the mixture, as shown in Fig. 2 b. As a comparison, Fig. 2 c displays a very good dispersion of LCs doped with MWCNTs as expectation when alcohol was applied. Fig. $2 \mathrm{~d}$ shows the result after three days later. The mixture was still clear, without any stratification. In this comparative measurement, the concentration of doping MWCNTs was $0.02 \%$ by weight at room temperature. The conclusion was drawn that the MWCNTs uniformly mixed with the LCs due to the use of alcohol.

The empty cells were manufactured by pairs of glass substrates coated with transparent ITO electrodes. The substrates were then coated with PI to ensure alignment for the LC materials. The cells composed of pristine nematic LC and nematic added with alcohol were fabricated. The system of measurement, EOT-01, was developed by the Changchun Institute of Optics, Fine Mechanics and 
Physics, Chinese Academy of Sciences. Following the steps of the system, a halogen lamp was used as a light source for characterizing the optical transmission properties. A function generator provided an AC electric field (square wave, frequency of $1 \mathrm{kHz}$, voltage of $0-10 \mathrm{~V})$ across the cell thickness. The threshold voltage and the driving voltage are defined as the voltages at which the transmitted light power is increased to $10 \%$ and $90 \%$ of the initial value at null voltage, respectively. In Fig. 3, the two curves of the optical transmittance-time for the two types of LC cells are shown. The purpose of this measurement was to observe the affect of alcohol as a dispersing solvent. From the two curves, the data of the optical transmittance were nearly similar. And no differences were observed between the curves. The measured results indicated that the alcohol utilized as a dispersing solvent did not affect the electro-optical properties of the two types of LC cells.

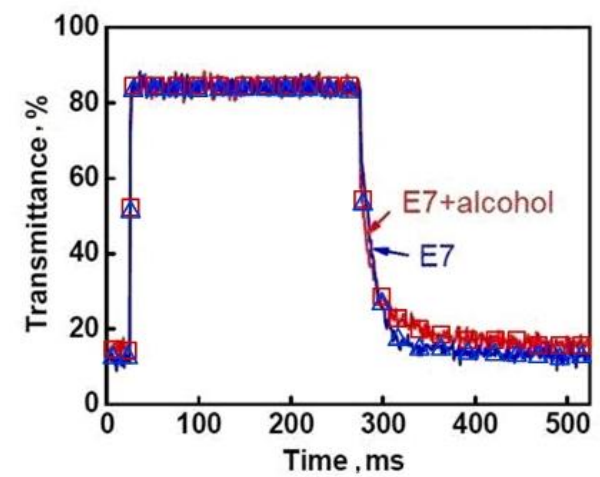

Fig. 3. Transmittance-time characteristics of LC cells: the blue circle curve represents the LC cell of pristine E7 without alcohol, and the red square curve shows the LC cell of nematic E7 with alcohol

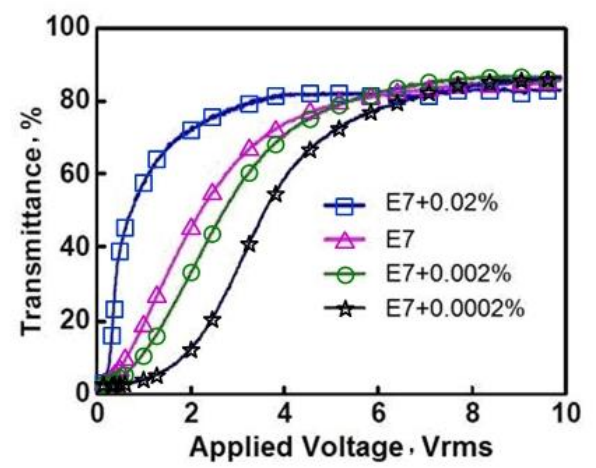

Fig. 4. Results of electro-optical features under various concentrations

In Fig. 4, the electro-optical transmissions of LC lens under various applied voltages are shown for the four types of cells. In comparative measurements, the four fabricated cells included the pristine LC, LC doped with MWCNTs at a concentration of $0.00002 \%$, LC doped with MWCNTs at a concentration of $0.0002 \%$, and LC doped with MWCNTs at a concentration of $0.002 \%$. The system of measurement, EOT-01, was utilized to measure the classic electro-optical characteristics of the fabricated LC cells. The other experimental setup was the same as the abovementioned experiment. The threshold voltage of LC cell is [15]:
$V_{t h}=\pi \sqrt{\frac{4 K_{11}-2 K_{22}+K_{33}}{4 \varepsilon_{0} \Delta \varepsilon}}$,

where $K_{i i}$ represents different kinds of elastic constant, and $\varepsilon$ is dielectric constant. Threshold voltage was determined by elastic constant and dielectric constant. The purpose of this measurement was to observe the electro-optical features under various concentrations of MWCNT doping. Consistent with the expectations, the different MWCNT doping concentrations strongly influenced the features of the LC lens. To measure the dielectric anisotropy, a LCZ meter (NF-2341) running an $\mathrm{AC}$ voltage of $50 \mathrm{mV}$ at $1 \mathrm{kHz}$ was used. The entire experimental system was interfaced with a PC via LabVIEW. With the LCZ meter, the dielectric anisotropy was measured, as presented in Table 1. The data indicate that the dielectric anisotropy of the mixture was relational to the MWCNT concentrations. When concentration of doped MWCNT is below $0.002 \%$, dielectric constant of LC has nearly no changes. However, the threshold voltage slightly starts to increase as elastic constant changes. While the concentration of doped MWCNT starts to rise to $0.02 \%$, the dielectric constant will significantly increase, and its threshold voltage immediately reduces. The results of Fig. 4 and Table 1 show that the different concentrations of doped MWCNT strongly influence electro-optical features of LC, which could effectively prove the former results about electricoptical features of the doped CNT into LC materials [11].

Table 1. Results of dielectric anisotropy under various concentrations doped with MWCNTs

\begin{tabular}{|c|c|}
\hline $\begin{array}{c}\text { Various concentrations doped with } \\
\text { MWCNT, wt.\% }\end{array}$ & $\begin{array}{c}\text { Dielectric } \\
\text { anisotropy }\end{array}$ \\
\hline 0.0 & 15.16 \\
\hline 0.0002 & 14.98 \\
\hline 0.002 & 15.93 \\
\hline 0.02 & 20.15 \\
\hline
\end{tabular}

Fig. 5 shows the response time of the LC lens doped with different concentrations of MWCNTs, including rise time of LC lens, and fall time of LC lens. In these measurements, those parameters respectively frequency and voltage of square wave signal, were $1 \mathrm{KHz}$ and $6 \mathrm{~V}$. The rise time of LC is [15]:

$$
\tau_{r}=\frac{\gamma_{1} d^{2}}{\varepsilon_{0} \Delta \varepsilon V^{2}-\pi^{2} K_{i i}},
$$

where elastics constant $K_{i i}$, dielectric anisotropy $\Delta \varepsilon$, and viscosity coefficient $\gamma_{1}$ affect the rise time simultaneously. Because of trace amount dopants, the viscosity coefficient of the mixture had no changes, which could be seen as a constant. And dielectric anisotropy had no changes under this condition, as presented in Table 1 . Those caused that the rise time slowly increased. Until to $0.02 \%$ concentrations, the dielectric constant started to rise, while the rise time started to drop, as shown in Fig. 5 and Table 1. It was clear that the dominant factor was the dielectric anisotropy rather than the elastic constant. That caused the rise time to apparently drop rather than the rise time of the conventional LC lens. The fall time of LC is [15]:

$\tau_{f}=\frac{\gamma_{1} d^{2}}{\pi^{2} K_{i i}}$, 
where elastics constant $K_{i i}$ and viscosity coefficient $\gamma_{1}$ affect the fall time at the same time. Based on the above analyses, the viscosity coefficient was seen as a constant because the concentrations of the dopants were far below $0.02 \%$, which are proved in the measurements. Therefore, the changes of fall time were only decided by elastic constant. Fig. 5 shows different traces under different proportions of MWCNTs. The reason, the shapes about all traces coincide with each other, is that the fabricated LC lens is also a LC device. The LC device has this shape of response time. Fig. 5 shows that the proper concentration of doped MWCNT can lead to some extent improvement on response time, which is another method to improve the basic features of LC lens. The dropping trend would be enhanced while the concentration started to increase. According to these results, to achieve fast focusing time and low operating voltage simultaneously, the perfect concentration of the designed LC lens was chosen as $0.02 \%$.

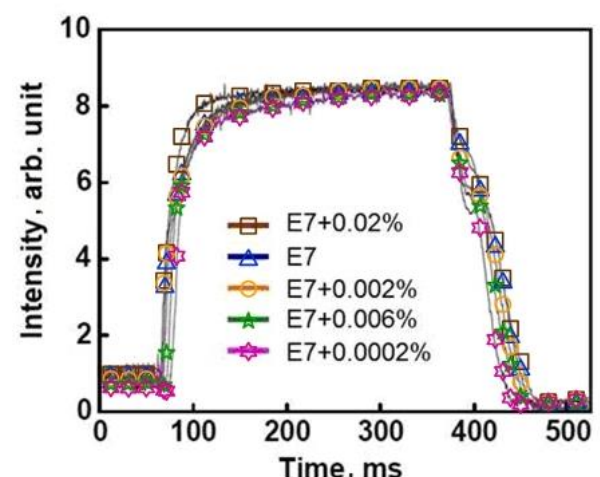

Fig. 5. Response time of LC cells doped under different concentrations, including rise time of LC lens, and fall time of LC lens

\section{CONCLUSIONS}

In the paper, the electro-optical properties of LC lens doped with MWCNTs were studied. The experiments showed that the dielectric anisotropy of the mixture was changed after doping. When the doping concentration of the mixture was less than $0.002 \%$, the dielectric constant was almost constant. However, if the doping concentration was $0.02 \%$, the dielectric constant started to rise rapidly, which improved the electro-optical properties of the LC lens. The experiment showed that the rise time and the threshold voltage value both decreased. However, due to the elasticity coefficient of the MWCNTs increasing with an increased doping concentration, the downward trend appeared during the fall time. In this way, the LC lens doped with $0.02 \%$ MWCNTs had a relatively faster response time than the conventional LC lens. This novel LC lens has great potential in many practical imaging applications and imaging commercialization.

\section{Acknowledgments}

This work was supported by a grant from the Research Foundation of Education Bureau of Hubei Province China (Q20141507), a grant from the Natural Science Foundation of Hubei Province of China (2014CFB771), China Postdoctoral Science Foundation (2014M562017), the
Science Foundation of the Wuhan Institute of Technology (X2014012, and K201545), and the Open Foundation for Hubei Key Laboratory of Intelligent Robot (HBIR 201405).

\section{REFERENCES}

1. Ren, H., Fox, D., Wu, S.-T. Liquid Crystal Lens with Large Focal Length Tunability and Low Operating Voltage Optics Express 15 (18) 2007: pp. $11328-11335$.

2. Ye, M., Wang, B., Uchida, M., Yanase, S., Takahashi, S., Sato, S. Focus Tuning by Liquid Crystal Lens in Imaging System Applied Optics 51 (31) 2012: pp. 7630-7635.

3. Spangenberg, D., Dudley, A., Neethling, P., Rohwer, E., Forbes, A. White Light Wavefront Control with a Spatial Light Modulator Optics Express 22 (11) 2014: pp. 13870-13879.

4. Hilario, P., Villangca, M., Tapang, G. Independent Light Fields Generated using a Phase-Only Spatial Light Modulator Optics Letter 39 (7) 2014: pp. 2036-2039. http://dx.doi.org/10.1364/OL.39.002036

5. Peng, Y., Li, H., Zhong, Q., Liu, X. Liquid-CrystalDisplay-Based Touchable Light Field Three-Dimensional Display using Display-Capture Mapping Calibration Applied Optics 51 (25) 2012: pp. 6014-6019. http://dx.doi.org/10.1364/AO.51.006014

6. Welker, D., Kuzyk, M. Suppressing Vibrations in a Sheet with a Fabry-Perot Photomechanical Device Optics Letter 22 (6) 1997: pp. 417-418. http://dx.doi.org/10.1364/OL.22.000417

7. Han, Z., Bozhevolnyi, S. Plasmon-Induced Transparency with Detuned Ultracompact Fabry-Perot Resonators in Integrated Plasmonic Devices Optics Express 19 (4) 2011: pp. 3251 - 3257.

8. Xu, S., Li, Y., Liu, Y., Sun, J., Ren, H., Wu, S.T. FastResponse Liquid Crystal Mcrolens Micromachines 5 (2) 2014: pp. 300-324.

9. Ren, H., Xu, S., Lin, Y., Wu, S.T. Adaptive-Focus Lenses Optics \& Photonics News 19 (10) 2008: pp. 42-47. http://dx.doi.org/10.1364/OPN.19.10.000042

10. Baughman, R.H., Zakhidov, A.A., Heer de, W.A. Carbon Nanotubes- the Route Toward Applications Science 297 (5582) 2002: pp. 787-792.

11. Lu, S., Chien, L. Carbon Nanotube Doped Liquid Crystal OCB Cells: Physical and Electro-Optical Properties Optics Express $16(17)$ 2008: pp. $12777-12785$. http://dx.doi.org/10.1364/OE.16.012777

12. Rajasekharan, R., Dai, Q., Wilkinson, T. Electro-Optic Characteristics of a Transparent Nanophotonic Device Based on Carbon Nanotubes and Liquid Crystals Applied Optics 49 (11) 2010: pp. 2099-2104.

13. Shriyan, S., Fontecchio, A. Analysis of Effects of Oxidized Multiwalled Carbon Nanotubes on Electro-Optic Polymer/Liquid Crystal Thin Film Gratings Optics Express 18 (24) 2010: pp. $24842-24852$. http://dx.doi.org/10.1364/OE.18.024842

14. Li, H., Pan, F., Wu, Y.T., Zhang, Y.D., Xie, X.L. Optical Study of Liquid Crystal Lens Doped with Multiwalled Carbon Nanotubes Materials Science (Medžiagotyra). (Accepted)

15. Gennes, P.G., de Prost, J. The Physics of Liquid Crystals (Second Edition). Clarendon, Oxford, 1995: pp. 198-263. 